American Journal of Biochemistry and Biotechnology 4 (2): 85-94, 2008

ISSN 1553-3468

(C) 2008 Science Publications

\title{
The Frequency of Polymorphisms affecting Lead and Mercury Toxicity among Children with Autism
}

\author{
Shannon Rose, Stepan Melnyk, Alena Savenka, Amanda Hubanks, Stefanie Jernigan \\ Mario Cleves and S. Jill James \\ University of Arkansas for Medical Sciences, College of Medicine, Department of Pediatrics \\ Arkansas Children's Hospital Research Institute, 1120 Marshall St., Little Rock, AR 72202
}

\begin{abstract}
Individual risk of developmental neurotoxicity with exposure to environmentally relevant levels of lead and mercury is likely to be determined by genetic susceptibility factors as well as additive interactions with other environmental pollutants, cumulative dose, and the developmental stage of exposure. The apparent increase in autism diagnosis over the last 15 years has enhanced interest in the possibility that an environmental trigger may be required to uncover the genetic liability in some cases of autism. The exquisite sensitivity of the developing brain and immune system to very low levels of lead and mercury give this hypothesis biologic plausibility. Delta aminolevulinic acid dehydratase (ALAD) and coproporphyin oxidase (CPOX) are two enzymes inhibited by low levels of lead and mercury, respectively. Common polymorphisms in these genes have been associated with elevated blood levels of lead and mercury and could potentially increase vulnerability to prenatal and/or postnatal developmental neurotoxicity. To explore this possibility, the frequency of the ALAD2 variant and variants in CPOX-4 and CPOX-5 were evaluated in 450 autistic children and 251 unaffected controls. A significant increase in the frequency of the ALAD2 allele was observed; however, contrary to our hypothesis, the frequency of both CPOX variants was significantly lower among the autistic children. Both lead and mercury induce oxidative stress by depleting the major intracellular antioxidant, glutathione. Among 242 autistic children with the variant ALAD2 allele, significant decreases in plasma glutathione and in the glutathione redox ratio were observed. These results suggest that children with autism who inherit the ALAD2 allele with lower glutathione levels may be at increased risk for lead toxicity during prenatal and postnatal neurodevelopment.
\end{abstract}

Key words: ALAD, CPOX, polymorphism, lead, toxicity, autism

\section{INTRODUCTION}

Autism is a complex neurodevelopmental disorder that is usually diagnosed in early childhood and is characterized by deficits in social reciprocity, impairments in verbal and non-verbal communication, and unusual behaviors that tend to be repetitive and hyper-focused. The diagnosis is based solely on behavioral criteria because there are no established genetic or biochemical biomarkers for autism. The biologic basis for autism is thought to be largely genetic due to the high concordance between monozygotic twins and the high recurrence rate within affected families $^{[1]}$. The fact that the twin concordance is less than $100 \%$ (60\% for severe autism and $90 \%$ for high functioning autism) suggests that additional environmental or epigenetic factors may be required to uncover the genetic liability ${ }^{[2,3]}$. Although it is well known that the developing human brain is much more sensitive to low level environmental neurotoxins than the adult brain, it is disturbing that the developmental toxicology of most of the industrial chemicals present in our environment has not yet been evaluated and published "safe" levels are often based on adult statistics $^{[4,5]}$. Moreover, it is possible that individual vulnerability to toxic environmental exposures varies according to additive and interactive effects of multiple genetic polymorphisms that negatively affect detoxification capacity.

Intracellular redox imbalance and oxidative stress is induced with chronic exposure to many common environmental contaminants including heavy metals, arsenic, polychlorinated biphenyls (PCBs), dioxin, transition metals, polycyclic aromatic hydrocarbons (PAHs), organochlorine, organophosphate pesticides, and trichloroethylene ${ }^{[6]}$. While chemically and

Corresponding Author: $\quad$ S. Jill James, Ph.D., Arkansas Children's Hospital Research Institute, 1120 Marshall St., Slot 512-40B, Little Rock, AR 72202 
structurally diverse, these common environmental toxicants all directly or indirectly deplete the major intracellular antioxidant glutathione, which is also essential for Phase II detoxification. Glutathione depletion shifts the intracellular redox balance towards unopposed oxygen free radical production and oxidative stress $^{[7]}$. Although exposure to individual chemicals may be at subtoxic concentrations, it is important to note that the multiple environmental chemicals present in air, soil, and food can interact additively or synergistically to induce oxidative stress in the developing brain at levels that do not affect adult brain. We and others have reported that children with autism have increased concentrations of biomarkers associated with oxidative stress ${ }^{[8]}$. Evidence for membrane lipid peroxidation, elevated urinary isoprostanes, and reduced activity of antioxidant enzymes have been reported ${ }^{[9-16]}$. Further, recent reports indicate that many autistic children have a significant decrease in plasma glutathione (GSH) levels, an increase in oxidized disulfide glutathione (GSSG), and a decrease in the glutathione redox ratio $(\mathrm{GSH} / \mathrm{GSSG})^{[8,17]}$. These results are consistent with inadequate glutathione synthesis or enhanced glutathione depletion in autistic children that could be genetically and/or environmentally influenced.

The 10-fold increase in the diagnosis of autism over the last decade has heightened interest in the possibility that an environmental trigger may be required to uncover the genetic predisposition in some cases of autism. The exquisite sensitivity of the developing brain and immune system to low level toxic exposures and the neurologic and immunologic dysfunction with autism give this hypothesis biologic plausibility ${ }^{[4]}$. In particular, the developmental neurotoxicity and immunotoxicity of environmentally relevant levels of lead and mercury is unequivocal ${ }^{[18-21]}$. In addition to glutathione depletion and oxidative stress, both lead and mercury have been shown to inhibit specific enzymes in the heme biosynthetic pathway that lead to the accumulation and excretion of specific porphyrin metabolites ${ }^{[22-24]}$. Figure 1 diagrams the sequential steps in heme biosynthesis, the porphyrin intermediates, and the specific enzymes inhibited by lead and mercury. The vital importance of this highly conserved pathway is due to the fact that heme is a component of several essential proteins such as hemoglobin for oxygen transport, the cytochrome p450 family for detoxification, mitochondrial cytochromes for ATP synthesis, and nitric oxide synthase for nitric oxide-mediated membrane signal transduction. Recently, significant porphyinuria was found in a cohort of autistic children ${ }^{[23]}$ and adds support to several reports of an association between heavy metal exposures and autism ${ }^{[25-27]}$.

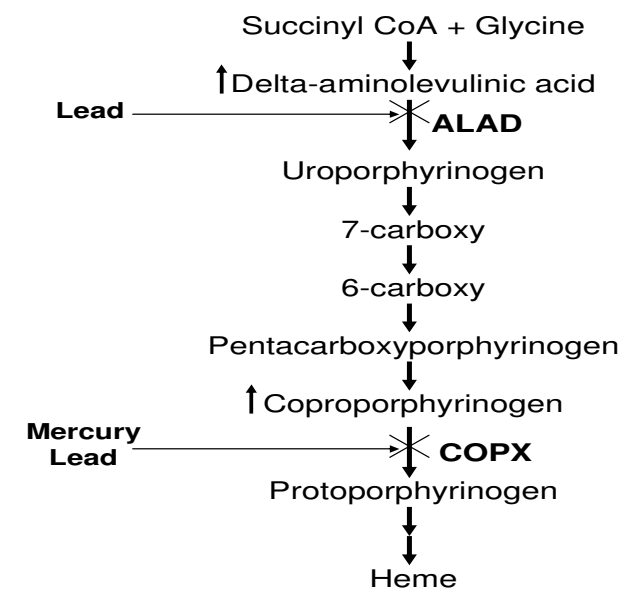

Fig. 1: Pathway of heme biosynthesis showing the several porphyrin intermediates and the inhibition of ALAD and CPOX enzymes by lead and mercury

Because the risk of developmental toxicity with low dose environmental chemicals is likely to be influenced by individual genetic determinants of susceptibility, it was of interest to evaluate autistic children for the frequency of polymorphic variants that affect the tissue distribution and toxicity of lead and mercury. A polymorphism in the delta aminolevulinic acid dehydratase (ALAD) gene has been associated with altered toxicokinetics of lead and elevated blood lead levels ${ }^{[28-30]}$. ALAD catalyzes the second step in heme biosynthesis and is strongly inhibited by lead as well as mercury, trichloroethylene and bromobenzine ${ }^{[31-}$ ${ }^{33]}$. The most common ALAD polymorphism is a G-to$\mathrm{C}$ transversion at position 177 in the coding region resulting in the substitution of asparginine for lysine at residue 59. The ALAD G177C (rs1800435) has two co-dominant alleles ALAD1 (177G) and ALAD 2 (177C) resulting in genotypes ALAD 1-1, heterozygous ALAD 1-2, and mutant ALAD 2-2.

Two polymorphisms in the coproporphyrinogen oxidase (CPOX) gene, CPOX-4 (rs1131857) and CPOX-5 (rs1729995), have been recently identified and may affect sensitivity to heavy metal toxicity ${ }^{[22,28]}$. CPOX is the sixth enzyme in the heme synthetic pathway and is inhibited by lead, mercury and arsenic as well as by chlorinated benzenes and alcohol ${ }^{[2,34]}$. Inhibition of CPOX results in excretion of a highly specific urinary porphyrin pattern consistent with inhibition of specific enzymes in the heme pathway by mercury ${ }^{[35,36]}$. The CPOX-4 A814C variant, resulting in an asparagine-to-histidine change at amino acid 227, has been associated with increased urinary excretion of a specific porphyrin (precoproporphyrin) and is 
Am. J. Biochem. \& Biotech., 4 (2): 85-94, 2008

proposed to be a biomarker of susceptibility to mercury toxicity ${ }^{[22]}$. The CPOX G $>$ A transition in exon 5 is a synonymous mutation (E330E) that has not been characterized functionally. Of related interest, low glutathione levels have been associated with an increase in urinary prophyrins ${ }^{[37,38]}$.

Based on these considerations and the possibility that a genetic susceptibility may underlie an increased sensitivity to heavy metal toxicity during neurodevelopment, we evaluated the prevalence of polymorphisms in the ALAD and CPOX genes in a cohort of children diagnosed with autistic disorder.

\section{MATERIALS AND METHODS}

Subjects: Participating children were referral patients recruited from the Dennis Developmental Center at the University of Arkansas for Medical Sciences and from the autism clinics of participating physicians in New York and Florida ${ }^{[8]}$. The diagnosis of autistic spectrum disorder was made using criteria defined by the Diagnostic and Statistical Manual of Mental Disorders, Fourth Edition (DSM-IV) and the Childhood Autism Rating Scales (CARS) or the Autism Diagnostic Observation Schedule (ADOS). Exclusion criteria included current infection or fever, chronic seizures, and rare genetic diseases associated with symptoms of autism (e.g., fragile X, Rett syndrome, tuberous sclerosis). A total of 451 case children participated in the genetic analysis of which 242 case children contributed a fasting blood sample for glutathione evaluation. The case children were $\sim 97 \%$ Caucasian with mental capacity ranging from moderate mental retardation to high functioning and an age range between $3-14$ years (mean + SD: $6.9 \pm 2.9$ ). The 75 control children for the metabolic analysis were healthy Caucasians with no history of chronic disease, autism, or other neurologic disorder who had participated as controls for similar metabolic studies of children with Down syndrome $^{[39]}$ and cystic fibrosis ${ }^{[40]}$. The mean age and SD of the control children was $10.8 \pm 4.1$ years. The protocol was approved by the Institutional Review Board of the University of Arkansas for Medical Sciences and parental written informed consent was obtained.

Genetic analysis: For the genetic analysis, an additional 176 unaffected controls (total control $\mathrm{n}=$ 251) consisted of participants in an ongoing study of congenital heart defect risk described previously ${ }^{[41]}$. Genomic DNA was extracted from the blood leukocytes using Puregene DNA Purification Kit (Gentra Systems, Inc., Minneapolis, MN). Genotyping was performed with allele-specific fluorescent primerprobe sets supplied by ABI Assays by Design (Applied Biosystems, Foster City, CA). Primer and probe sequences for ALAD (SNP500 Cancer ${ }^{[42]}$ ) and CPOX SNPs $^{[28]}$ are listed in Table 1. PCR reactions were carried out with ABI PRISM 7700 Sequence Detection Systems under the following thermal cycling conditions: one cycle at $95^{\circ} \mathrm{C}$ for $10 \mathrm{~min}$ (Taq activation), followed by 40 cycles of $92^{\circ} \mathrm{C}$ for $15 \mathrm{sec}$ (denature) and $60^{\circ} \mathrm{C}$ for $1 \mathrm{~min}$ (anneal/extend). The reaction components were as follows: $900 \mathrm{nM}$ of each primer, $200 \mathrm{nM}$ each probe, 1X of TaqMan Universal Master Mix (\#4324018 ABI, Foster City, CA), and 50 ng genomic DNA. Utilizing data collected from a previous study ${ }^{[8]}$, we conducted a gene-gene interaction analysis between the ALAD 2 allele and the G allele of the reduced folate carrier (RFC1) 66A>G polymorphism that we previously showed to be significantly elevated among autistic children. The primers used for RFC1 are listed in Table 1. Because not every DNA sample amplified, there are slight variations in the total $n$ between the different polymorphisms.

Metabolic analysis: Fasting blood samples were collected from 242 autistic and 75 unrelated control children into EDTA-Vacutainer tubes and immediately chilled on ice before centrifuging at $4000 \mathrm{x} \mathrm{g}$ for 10 min at $4^{\circ} \mathrm{C}$. Plasma aliquots were transferred into cryostat tubes and stored at $-80^{\circ} \mathrm{C}$ until extraction and HPLC quantification. All samples were analyzed within one month of receipt. For determination of total glutathione, $50 \mu \mathrm{L}$ freshly prepared $1.43 \mathrm{~mol} \mathrm{~L}^{-1}$ sodium borohydride solution containing $1.5 \mu \mathrm{mol} \mathrm{L} \mathrm{L}^{-1}$ EDTA, $66 \mathrm{mmol} \mathrm{L}^{-1} \mathrm{NaOH}$ and $10 \mu \mathrm{L}$ isoamyl alcohol was added to $200 \mu \mathrm{L}$ plasma to reduce all sulfhydryl bonds and incubated at $40^{\circ} \mathrm{C}$ in a shaker for $30 \mathrm{~min}$. To precipitate proteins, $250 \mu \mathrm{L}$ ice cold $10 \%$ metaphosphoric acid was added, mixed well, and the sample was incubated for an additional $30 \mathrm{~min}$ on ice. After centrifugation at $18,000 \times \mathrm{g}$ for $15 \mathrm{~min}$ at $4{ }^{\circ} \mathrm{C}$, the supernatant was filtered through a $0.2 \mu \mathrm{m}$ nylon membrane filter (PGC Scientific, Frederic, MD) and a $20 \mu \mathrm{L}$ aliquot was injected into the HPLC system.

For determination of free reduced glutathione and oxidized disulfide glutathione (GSSG), $100 \mu \mathrm{L}$ of $10 \%$ meta-phosphoric acid was added to $200 \mu \mathrm{L}$ plasma to precipitate protein; the solution was mixed well and incubated on ice for $30 \mathrm{~min}$. After centrifugation for 15 min at $18,000 \mathrm{~g}$ at $4^{\circ} \mathrm{C}$, supernatants were passed through a $0.2 \mu \mathrm{m}$ nylon membrane filter and $20 \mu \mathrm{L}$ was injected into the HPLC system.

The separation of metabolites was performed using HPLC with a Shimadzu solvent delivery system (ESA model 580) and a reverse phase $\mathrm{C}_{18}$ column $(5 \mu \mathrm{m} ; 4.6$ x $150 \mathrm{~mm}$, MCM, Inc., Tokyo, Japan) obtained from ESA, Inc. (Chemsford, MA). A $20 \mu \mathrm{L}$ aliquot of plasma extract was directly injected onto the column using Beckman Autosampler (model 507E). All plasma metabolites were quantified using a model 5200A using Beckman Autosampler (model 507E). All plasma metabolites were quantified using a model 5200A Coulochem II and CoulArray electrochemical detection systems (ESA, Inc., Chelmsford, MA) equipped with a dual analytical cell (model 5010), a 4-channel analytical cell (model 6210) and a guard cell (model 5020). The concentrations of glutathione metabolites were 
calculated from peak areas and standard calibration curves using HPLC software.

Table 1: Primers and TaqMan probes used for genotyping

\begin{tabular}{ll}
\hline SNP (rs no.), primer/probe & 5'-3' sequence \\
\hline ALAD K68N; 177G>C (rs1800435) & \\
Forward & TGCCTTCCTTCAACCCCTCTA \\
Reverse & CAAGGGCCTCAGCATCTCTT \\
G-allele & 6FAM-TGTGAAGCGGCTGG-MGB \\
C-allele & VIC-TGTGAACCGGCTGG-MGB \\
\hline CPOX exon 4: N272H; 814A>C (rs1131857) & \\
Forward & CCA GTA ATG CTG AAT CTC AAA AGT CC \\
Reverse & GGA CAG CGT CTT CTT GAT TCA AGT AT \\
A-allele* & 6FAM-ACC ACT GCT TGT TGC CTA CCA AAT CA-TAMRA \\
C-allele* & VIC-ACC ACT GCT TGT GGC CTA CCA AAT C-TAMRA \\
\hline CPOX exon 5: E330E; G>A (rs1729995) & \\
Forward & \\
Reverse & GCT GTG TTT TCC AGG TGT GAT GA \\
G-allele* & GGA ATG TAA GAA GGA ACT ACA GCC CT \\
A-allele* & VIC-CCC CGC CGC TCT CCA CG-TAMRA \\
\hline$R F C-180 G>A$ & 6FAM-CCC CGC CGT TCT CCA CGA T-TAMRA \\
Forward & \\
Reverse & \\
G-Probe 1 & GGCCTGACCCCG AGCT \\
A-Probe 2 & AGCCGTAGAAGCAAAGGTAGCA \\
\hline
\end{tabular}

*reverse compliment

Statistical analysis: The data were prospectively collected and analyzed using SigmaStat software. Metabolic data are presented as the means \pm SD. Statistical differences between case and control children were determined using the Student's t test with significance set at 0.05 . For the genotype analysis, odds ratios and $95 \%$ confidence intervals were calculated using unconditional logistic regression models and tested using chi square analysis. Cases and controls were tested for Hardy-Weinberg equilibrium using the exact test implemented in STATA GENHW command $^{[43]}$. Gene-gene interactions were tested by including appropriate pair-wise indicator variables into unconditional logistic regression models. However, if corrected for multiple comparisons, statistical significance was lost.

\section{RESULTS AND DISCUSSION}

Genotyping: The control allele frequencies for the 3 genotypes were within the range of previously published reports and were all in Hardy Weinberg equilibrium $^{[28,44]}$. The results presented in Table 2 indicate that the $A L A D-1 \quad \mathrm{C}$ allele frequency was significantly elevated among autistic children with an odds ratio of $1.66(\mathrm{p}=0.02)$. This primarily reflected the heterozygous ALAD 1-2 variant since the homozygous $A L A D 2-2$ variant is quite rare in the general population. The frequency of the ALAD1-2 heterozygote was $20 \%$ among autistic children compared to $13 \%$ in controls indicating the children with the $A L A D 177 \mathrm{CG}$ genotype were approximately
1.7 times more likely to be autistic (OR: $1.67 ; \mathrm{p}=$ 0.02). Because of the low frequency of the $A L A D 2-2$ genotype, $A L A D$ 1-2 and 2-2 were combined and compared with the ALAD 1-1 genotype. Subjects harboring either the 1-2 or 2-2 genotype had a 1.67-fold increased risk of being autistic.

In contrast, the $\mathrm{C}$ allele frequency of the $C P O X$ exon $4814 \mathrm{~A}>\mathrm{C}$ variant was highly under-represented among autistic children compared to controls (Table 3). The odds ratio for the homozygous $814 \mathrm{CC}$ variant was 0.29 ( $\mathrm{p}=0.007)$ whereas the combined wildtype normal AA and heterozygous variants were overrepresented with an odds ratio of 3.36 ( $\mathrm{p}=0.007$ ). Similarly, the frequency of the $C P O X$ exon $5 \mathrm{G}>\mathrm{A}$ variant was significantly decreased among autistic children (Table 3 ). The odds ratio for the homozygous AA variant was $0.54(\mathrm{p}=0.03)$ with marginal significance for the heterozygous $\mathrm{AG}$ genotype $(\mathrm{p}=$ 0.08). The combined $\mathrm{AA}$ and $\mathrm{AG}$ variants were significantly under-represented among autistic children (OR: $0.70 ; \mathrm{p}=0.03$ ). The wildtype normal genotypes of both $C P O X$ exon 4 and 5 were significantly more prevalent suggesting that these genotypes were protective.

Significant gene-gene interactions were found between the $A L A D 177 \mathrm{C}>\mathrm{G}$ polymorphism and the reduced folate carrier $R F C 180 \mathrm{~A}>\mathrm{G}$ variant as shown in Table 4. In a previously published univariate analysis $^{[8]}$, we demonstrated that the frequency of the RFC1 $80 \quad \mathrm{G}$ allele was significantly increased among 
Am. J. Biochem. \& Biotech., 4 (2): 85-94, 2008

Table 2: ALAD K68N; 177G >C (rs1800435)

\begin{tabular}{|c|c|c|c|c|}
\hline Genotype & Cases N (\%) & Controls N (\%) & OR $(95 \% \mathrm{CI})$ & p-value \\
\hline G allele & $794(89.2 \%)$ & $459(92.9 \%)$ & Reference & \\
\hline $\mathrm{C}$ allele & $96(10.8 \%)$ & $35(7.1 \%)$ & $1.60(1.05,2.45)$ & 0.024 \\
\hline ALAD-1 (GG) & $352(79.1 \%)$ & $213(86.2 \%)$ & Reference & \\
\hline ALAD 1-2 (GC) & $90(20.2 \%)$ & $33(13.4 \%)$ & $1.65(1.05,2.63)$ & 0.023 \\
\hline ALAD 2-2 (CC) & $3(0.7 \%)$ & $1(0.4 \%)$ & $1.82(0.14,95.73)$ & 0.60 \\
\hline ALAD 1-2/2-2 (GC+CC) & $93(20.9 \%)$ & $34(13.8 \%)$ & $1.66(1.06,2.62)$ & 0.020 \\
\hline \multicolumn{5}{|c|}{ Table 3: $\quad$ CPOX exon 4 N272H; $177 \mathrm{~A}>\mathrm{C} \quad(\mathrm{rs} 1131857)$} \\
\hline Genotype & Cases N (\%) & Controls N (\%) & OR $(95 \% \mathrm{CI})$ & p-value \\
\hline A allele & $778(88.8 \%)$ & $428(85.3 \%)$ & Reference & \\
\hline $\mathrm{C}$ allele & $98(11.2 \%)$ & $74(14.7 \%)$ & $0.73(0.52,1.02)$ & 0.054 \\
\hline AA & $347(79.2 \%)$ & $190(75.7 \%)$ & Reference & \\
\hline $\mathrm{AC}$ & $84(19.2 \%)$ & $48(19.1 \%)$ & $0.96(0.63,1.46)$ & 0.832 \\
\hline $\mathrm{CC}$ & $7(1.6 \%)$ & $13(5.2 \%)$ & $0.29(0.10,0.81)$ & 0.007 \\
\hline $\mathrm{AA}+\mathrm{AC}$ & $431(98.4 \%)$ & $238(94.8 \%)$ & $3.36(1.23,10.0)$ & 0.007 \\
\hline \multicolumn{5}{|c|}{ CPOX exon 5 E330E; G>A $\quad$ (rs1729995) } \\
\hline Genotype & Cases N (\%) & Controls N (\%) & OR $(95 \% \mathrm{CI})$ & p-value \\
\hline G allele & $662(73.7 \%)$ & $338(67.3 \%)$ & Reference & \\
\hline A allele & $236(26.3 \%)$ & $167(32.7 \%)$ & $0.73(0.58,0.94)$ & 0.011 \\
\hline GG & $245(54.6 \%)$ & $115(45.8 \%)$ & Reference & \\
\hline GA & $172(38.3 \%)$ & $108(43.0 \%)$ & $0.75(0.53,1.05)$ & 0.080 \\
\hline AA & $32(7.1 \%)$ & $28(11.2 \%)$ & $0.54(0.30,0.97)$ & 0.025 \\
\hline $\mathrm{GA}+\mathrm{AA}$ & $204(45.4 \%)$ & $136(54.2 \%)$ & $0.70(0.51,0.97)$ & 0.026 \\
\hline
\end{tabular}

autistic children. Relative to controls, the autistic children exhibited significantly increased frequency of the RFC-1 homozygous $80 \mathrm{GG}$ (33\% vs. 26\%) and heterozygous $80 \mathrm{GA}$ (52\% vs. $41 \%)$. Children with either the $R F C-1$ AG or GG genotypes were approximately 2 times more likely to be autistic (OR: 2.26 and 1.96, respectively; $\mathrm{p}<0.001)$. In the present analysis, children with the compound heterozygous ALAD 177CG/RFC1 80AG genotype (2 mutant alleles) were almost 4-fold more likely to be autistic compared with unaffected controls (OR: 3.98; p = 0.001) suggesting an additive interaction between these two genotypes. Children with both the $A L A D$ homozygous GG genotype and RFC heterozygous or homozygous variant genotypes were 2.7-fold and 2.6 fold more likely to be autistic ( $\mathrm{p}=0.0002$ and 0.001 , respectively). Gene-gene interactions were also observed between the $A L A D 177 \mathrm{CG}$ variant and both $C P O X$ exon 4 and exon 5 genotypes. Children with both the $A L A D$ heterozygous genotype and the wildtype normal $C P O X$ exon 4 or exon 5 genotypes were 1.6 and 2-fold more likely to be autistic ( $\mathrm{p}=0.04$ and 0.02 , respectively).

To determine whether the $A L A D$ variant genotypes had a functional impact among autistic children, plasma free glutathione levels (GSH), oxidized disulfide glutathione (GSSG), and the redox ratio (GSH/GSSG) were compared between children with the wildtype GG genotype and children with either the heterozygous CG or homozygous CC variant genotypes. The results in Table 5 indicate that the children with the variant ALAD CG and CC genotypes had significantly lower concentrations of plasma total (free plus protein-bound) and free glutathione levels compared to the wildtype normal GG genotype. Levels of GSSG were not affected by ALAD genotype among autistic children.

The potential developmental neurotoxicity of heavy metals that are commonly encountered in the environment is a major public health concern and challenge because their identification and regulation could lead to preventive measures. Grandjean and Landrigan elegantly raised this issue in a recent review and provided evidence that the multitude of untested and unregulated chemicals present in drinking water, soil, and air could be contributing to a "silent pandemic" of neurodevelopmental disorders in modern society $^{[5]}$. For example, environmentally relevant levels of lead have been shown to be associated with deficits in cognition and development that translate into lifelong aberrations in attention, impulsivity, aggressiveness as well as impaired IQ, memory and language skills ${ }^{[45-48]}$. Additive and synergistic interactions between the chemicals plus variation in duration, timing, and dose during critical windows of development complicate retrospective evaluation of neurodevelopmental toxicity ${ }^{[49]}$. Prospective evaluation of maternal and 
cord blood as proposed in the National Children's Study could provide valuable information on early toxic exposures and subsequent risk of developmental disabilities. An alternative approach is the evaluation of genetic polymorphisms that could confer differential susceptibility to specific environmental exposures.

Increased vulnerability to environmentally relevant levels of lead and mercury due to individual genetic variation is a plausible hypothesis that could potentially result in neurodevelopmental toxicity and increase risk of developing autism. Both lead and mercury induce oxidative damage by depleting intracellular glutathione, the major intracellular mechanism for heavy metal detoxification $^{[50,51]}$. In addition, both lead and mercury inhibit enzymes in the heme biosynthetic pathway and have been shown to increase the excretion of abnormal porphyrins in the urine ${ }^{[23,32,36]}$. In this study, we evaluated the prevalence of two polymorphisms in the heme biosynthetic pathway that have previously been associated with lead and mercury toxicity ${ }^{[23,28]}$. The results were positive for the $A L A D$ polymorphism but negative for both of the $C P O X$ polymorphisms. To our knowledge, this is the first study to evaluate the prevalence of these environmentally relevant polymorphisms in autistic children.

Our results suggest that children carrying the $A L A D$-2 allele (ALAD 1-2 or 2-2) may have an increased susceptibility to lead toxicity that could contribute, in part, to the risk of developing autism. Higher concentrations of blood lead have been reported in subjects with the $A L A D 1-2$ genotype ${ }^{[29,52]}$ and also independently in children with autism ${ }^{[53]}$. Consistent with our hypothesis, the higher lead levels in children with autism may reflect, in part, an increase in the frequency of the $A L A D-2$ allele among these children. Lead tissue kinetics are altered in $A L A D-2$ carriers by decreasing lead uptake into cortical bone and increasing mobilization from trabecular bone ${ }^{[54,55]}$; thus, the increase in blood lead levels may reflect enhanced release of lead from bone. Further, it has been suggested that ALAD2 protein binds lead more tightly to the enzyme than ALAD1 resulting in a stronger inactivation of ALAD and increased risk of neurotoxicity in ALAD2 carriers $^{[30]}$. ALAD activity is inhibited by lead in a dose-response manner and the accumulation of the enzyme precursor, deltaaminolevulinic acid, may contribute to the neurotoxic effects of lead ${ }^{[55]}$. Recently, an association between elevated lead and decreased creatinine clearance among ALAD2 carriers suggested a negative effect of the polymorphism on renal function ${ }^{[54]}$.

Lead and mercury have a high affinity for the cysteine sulfhydryl (-SH) group of glutathione and form a heavy metal-glutathione conjugate that is subsequently metabolized and excreted in the bile and urine. In this way, glutathione serves as the body's natural chelator and provides the major mechanism of heavy metal detoxification and elimination. Chronic heavy metal exposure, however, can increase the requirement for de novo glutathione synthesis and lead to a decrease in cysteine and glutathione levels ${ }^{[56]}$. We previously showed that many children with autism have significantly lower cysteine and glutathione levels compared to unaffected controls ${ }^{[8]}$. The results of the present study indicate that autistic children carrying the $A L A D 2 \mathrm{C}$ allele had significantly lower GSH levels and GSH/GSSG redox ratio compared to children with $A L A D 1 \mathrm{G}$ allele. Because GSH levels have been shown not to change significantly in healthy children within this age range ${ }^{[57]}$, we do not think this difference can be attributed to the age difference between groups. Of related interest, elevation in blood lead levels reduces the antioxidant activity of paraoxonase-1 (PON 1), an enzyme that participates in the detoxification of organophosphates $^{[58]}$. The activity of PON-1 has been reported to be reduced in autistic children ${ }^{[59]}$.

The exon 4 CPOX variant has been previously associated with increased mercury levels ${ }^{[28]}$ and mercury-specific porphyrin patterns in autistic children $^{[23]}$. However, in the present study, the frequency of both the CPOX exon 4 and exon 5 variants was significantly decreased among autistic children. These results suggest that mercury-specific porphyrin patterns, if present in autistic children, most likely reflects heavy metal exposure that is independent of the $C P O X$ susceptibility alleles. Although $C P O X-4$ has been most extensively studied because of the amino acid substitution in the enzyme protein, it is interesting that the less studied $C P O X-5$ variant was similarly under-represented in autistic children. The CPOX-5 variant is a synonymous polymorphism that does not result in an amino acid change in the enzyme. However, several recent reports have suggested that "silent" genetic variations may have functional impact through inefficient RNA splicing and folding ${ }^{[60]}$. Thus, the $C P O X-5$ synonymous variant could be functional and an association with potential heavy metal toxicity should be further investigated.

Subtle alterations in gene expression that interact to disrupt a common pathway can induce a chronic metabolic imbalance that may contribute to complex genetic disease. As shown in Table 4, an additive interaction between the $A L A D-2$ allele and the $R F C l \mathrm{G}$ allele was found; i.e., the odds ratio with the combined genotypes was greater than either genotype alone. Specifically, children harboring the compound 
Am. J. Biochem. \& Biotech., 4 (2): 85-94, 2008

Table 4: Gene-gene interactions

\begin{tabular}{|c|c|c|c|c|}
\hline Genotype* & Cases & Controls & OR $(95 \% \mathrm{CI})$ & $\mathrm{p}$-value \\
\hline \multicolumn{5}{|l|}{ ALAD G>C / RFC1 G>A } \\
\hline ALAD / RFC1 GG/AA & $41(11.7 \%)$ & $42(26.6 \%)$ & Reference & \\
\hline ALAD / RFC1 & $11(3.1 \%)$ & $5(3.2 \%)$ & $2.25(0.72,7.06)$ & 0.162 \\
\hline ALAD / RFC1 & $35(10.0 \%)$ & $9(5.7 \%)$ & $3.98(1.70,9.32)$ & 0.001 \\
\hline ALAD / RFC1 & $14(4.0 \%)$ & $8(5.1 \%)$ & $1.79(0.68,4.73)$ & 0.237 \\
\hline \multicolumn{5}{|l|}{ ALAD G $>\mathrm{C} / \mathrm{CPOXex} 4 \mathrm{~A}>\mathrm{C}$} \\
\hline ALAD / CPOX ex4 GG/AA & $265(61.2 \%)$ & $158(64.0 \%)$ & Reference & \\
\hline ALAD / CPOX ex4 & $75(17.3 \%)$ & $27(10.9 \%)$ & $1.66(1.02,2.68)$ & 0.0402 \\
\hline \multicolumn{5}{|l|}{ ALAD G>C / CPOXex5 G>A } \\
\hline ALAD / CPOX ex5 GG/GG & $189(42.7 \%)$ & $99(40.1 \%)$ & Reference & \\
\hline ALAD / CPOXex 5 & $53(12.0 \%)$ & $13(5.3 \%)$ & $2.14(1.11,4.11)$ & 0.0229 \\
\hline
\end{tabular}

Table 5: Glutathione levels by ALAD genotype

\begin{tabular}{lllll}
\hline & $\begin{array}{l}\text { Control } \\
\mathrm{n}=75\end{array}$ & $\begin{array}{l}\text { Autistic }^{1} \\
\text { ALAD GG n=195 }\end{array}$ & $\begin{array}{l}\text { Autistic }^{1} \\
\text { ALAD CG+CC n=47 }^{2}\end{array}$ & $\mathrm{p}^{- \text {value }^{2}}$ \\
\hline Total GSH (tGSH) & $7.5 \pm 1.7$ & $5.69 \pm 1.3$ & $5.08 \pm 1.3$ & 0.007 \\
Free GSH (fGSH) & $2.2 \pm 0.9$ & $1.60 \pm 0.5$ & $1.4 \pm 0.4$ & 0.02 \\
GSSG & $0.24 \pm 0.1$ & $0.32 \pm 0.12$ & $0.32 \pm 0.13$ & ND \\
tGSH/GSSG & $28.2 \pm 7.0$ & $20.45 \pm 8.7$ & $17.4 \pm 5.7$ & 0.03 \\
fGSH/GSSG & $7.9 \pm 3.5$ & $5.64 \pm 2.6$ & $4.81 \pm 1.8$ & 0.05 \\
\hline
\end{tabular}

${ }^{1}$ Values from autistic children were all significantly different from controls with $p<0.001$

${ }^{2} \mathrm{p}$ values compare autistic children with ALAD wildtype GG genotype vs. ALAD variants $\mathrm{AG}+\mathrm{CC}$

$\mathrm{ND}=$ Not significantly different

heterozygous ALAD1-2/RFC1 CG genotype were almost 4 times more likely to be autistic. The $R F C-1$ polymorphism decreases the availability of folate methyl groups that provide precursors for glutathione synthesis $^{[8]}$. Theoretically, gene-gene interaction between the RFCl and $A L A D-2$ variants could be functionally related to the significant decrease in glutathione levels observed in the carriers of the $A L A D$ 2 allele. Although not additive, significant gene-gene interactions were also observed between the $A L A D$ 1-2 genotype and the $C P O X-4$ and $C P O X-5$ wildtype genotypes.

In summary, we have demonstrated that some autistic children have a significant increase in the frequency of the $A L A D 2$ variant allele that may underlie differential susceptibility to lead toxicity in these children. These data support the possibility that a genetic predisposition may increase vulnerability to lead toxicity during critical windows of prenatal and post-natal neurodevelopment. Although the data support a mechanistic contribution of glutathione depletion with the $A L A D-2$ variant, the contribution of decreased synthesis of essential heme proteins for detoxification and ATP synthesis cannot be ruled out. The measurement of blood lead levels (\% plasma/blood lead) in $A L A D-2$ positive autistic children would strengthen these possibilities. Clearly, the results of this study should be considered preliminary until confirmed in additional cohorts of autistic children.

Based on the behavioral diagnosis of autism, the brain is a common target for investigation into the biologic basis of autism. Nonetheless, because glutathione and heme proteins are ubiquitously expressed in all cells beyond the brain, research into the broader systemic effects of oxidative stress and heavy metal toxicity in autistic children should be pursued.

\section{REFERENCES}

1. Bailey, A., A. Le Couteur, I. Gottesman, P. Bolton, E. Simonoff, E. Yuzda, and M. Rutter, 1995. Autism as a strongly genetic disorder: evidence from a British twin study. Psychol. Med., 25: 6377.

2. London, E.A., 2000. The environment as an etiologic factor in autism: a new direction for research. Environ. Health Perspect., 108 (Suppl. 3): 401-404.

3. Lawler, C.P., L.A. Croen, J.K. Grether and J. Van de Water, 2004. Identifying environmental contributions to autism: Provocative clues and false leads. Ment. Retard. Dev. Disabil. Res. Rev., 10: 292-302.

4. Stein, J., T. Schettler, D. Wallinga, and M. Valenti, 2002. In harm's way: Toxic threats to child development. J. Dev. Behav. Pediatr., 23: S13-S22. 
5. Grandjean, P. and P.J. Landrigan, 2006. Developmental neurotoxicity of industrial chemicals. Lancet, 368: 2167-2178.

6. Li, Z., T. Dong, C. Proschel, and M. Noble, 2007. Chemically diverse toxicants converge on Fyn and c-Cbl to disrupt precursor cell function. PLoS. Biol., 5: e35.

7. Han, D., R. Canali, D. Rettori, and N. Kaplowitz, 2003. Effect of glutathione depletion on sites and topology of superoxide and hydrogen peroxide production in mitochondria. Mol. Pharmacol., 64: 1136-1144.

8. James, S.J., S. Melnyk, S. Jernigan, M.A. Cleves, C.H. Halsted, D.J. Wong, P. Cutler, M. Boris, K. Bock, J.J. Bradstreet, S.B. Baker and D.W. Gaylor, 2006. Metabolic endophenotype and related genotypes are associated with oxidative stress in children with autism. Am. J. Med. Genet., 141B: 947-956.

9. Ming, X., T.P. Stein, M. Brimacombe, W.G. Johnson, G.H. Lambert and G.C. Wagner, 2005. Increased excretion of a lipid peroxidation biomarker in autism. Prostaglandins Leukot. Essent. Fatty Acids, 73: 379-384.

10. Yorbik, O., A. Sayal, C. Akay, D. I. Akbiyik, and T. Sohmen, 2002. Investigation of antioxidant enzymes in children with autistic disorder. Prostaglandins Leukot. Essent. Fatty Acids, 67: 341-343.

11. Chauhan, A. and V. Chauhan, 2006. Oxidative stress in autism. Patholphysiology, 13: 171-181.

12. Kern, J.K. and A.M. Jones, 2006. Evidence of toxicity, oxidative stress, and neuronal insult in autism. J. Toxicol. Environ. Health B Crit. Rev., 9: 485-499.

13. Sogut, S., S.S. Zoroglu, H. Ozyurt, Y.H. Ramazan, F. Ozugurlu, E. Sivasli, O. Yetkin, M. Yanik, H. Tutkun, H. A. Savas, M. Tarakcioglu, and O. Akyol, 2003. Changes in nitric oxide levels and antioxidant enzyme activities may have a role in the pathophysiological mechanisms involved in autism. Clin. Chim. Acta, 331: 111-117.

14. Zoroglu, S.S., F. Armutcu, S. Ozen, A. Gurel, E. Sivasli, O. Yetkin, and I. Meram, 2004. Increased oxidative stress and altered activities of erythrocyte free radical scavenging enzymes in autism. Eur. Arch. Psychiatry Clin. Neurosci., 254: 143-147.

15. Yao, Y., W.J. Walsh, W.R. McGinnis and D. Pratico, 2006. Altered vascular phenotype in autism: Correlation with oxidative stress. Arch. Neurol., 63: 1161-1164.

16. McGinnis, W.R., 2004. Oxidative stress in autism. Altern. Ther. Health Med., 10: 22-36.
17. James, S.J., P. Cutler, S. Melnyk, S. Jernigan, L. Janak, D.W. Gaylor and J.A. Neubrander, 2004. Metabolic biomarkers of increased oxidative stress and impaired methylation capacity in children with autism. Am. J Clin. Nutr., 80: 1611-1617.

18. Costa, L.G., M. Aschner, A. Vitalone, T. Syversen and O.P. Soldin, 2004. Developmental neuropathology of environmental agents. Annu. Rev. Pharmacol. Toxicol., 44: 87-110.

19. Shanker, G. and M. Aschner, 2003. Methylmercury-induced reactive oxygen species formation in neonatal cerebral astrocytic cultures is attenuated by antioxidants. Mol. Brain Res., 110: 85-91.

20. Davidson, P.W., G.J. Myers, and B. Weiss, 2004. Mercury exposure and child development outcomes. Pediatr., 113: 1023-1029.

21. Sanfeliu, C., J. Sebastia, R. Cristofol and E. Rodriguez-Farre, 2003. Neurotoxicity of organomercurial compounds. Neurotox. Res., 5: 283-305.

22. Woods, J.S., D. Echeverria, N.J. Heyer, P.L. Simmonds, J. Wilkerson and F.M. Farin, 2005. The association between genetic polymorphisms of coproporphyrinogen oxidase and an atypical porphyrinogenic response to mercury exposure in humans. Toxicol. Appl. Pharmacol., 206: 113-120.

23. Nataf, R., C. Skorupka, L. Amet, A. Lam, A. Springbett and R. Lathe, 2006. Porphyrinuria in childhood autistic disorder: Implications for environmental toxicity. Toxicol. Appl. Pharmacol., 214: 99-108.

24. Beri, R. and R Chandra, 1993. Chemistry and biology of heme. Effect of metal salts, organometals and metalloporphyrins on heme synthesis and catabolism, with special reference to clinical implications and interactions with cytochrome P-450. Drug Metab. Rev., 25: 49-152.

25. Palmer, R.F., S. Blanchard, Z. Stein, D. Mandell and C. Miller, 2006. Environmental mercury release, special education rates, and autism disorder: an ecological study of Texas. Health Place., 12: 203-209.

26. Windham, G.C., L. Zhang, R. Gunier, L.A. Croen and J.K. Grether, 2006. Autism spectrum disorders in relation to distribution of hazardous air pollutants in the san francisco bay area. Environ. Health Perspect., 114: 1438-1444.

27. Bernard, S., A. Enayati, H. Roger, T. Binstock and L. Redwood, 2002. The role of mercury in the pathogenesis of autism. Mol. Psychiat., 7 (Suppl. 2): S42-S43. 
28. Woods, J.S., D. Echeverria, N.J. Heyer, P.L. Simmonds, J. Wilkerson and F.M. Farin, 2005. The association between genetic polymorphisms of coproporphyrinogen oxidase and an atypical porphyrinogenic response to mercury exposure in humans. Toxicol. Appl. Pharmacol2, 206: 113-120.

29. Montenegro, M.F., F. Barbosa Jr., V.C. Sandrim, R.F. Gerlach and J.E. Tanus-Santos, 2006. A polymorphism in the delta-aminolevulinic acid dehydratase gene modifies plasma/whole blood lead ratio. Arch. Toxicol., 80: 394-398.

30. Astrin, K.H., D.F. Bishop, J.G. Wetmur, B. Kaul, B. Davidow and R. J. Desnick, 1987. deltaAminolevulinic acid dehydratase isozymes and lead toxicity. Ann. N.Y. Acad. Sci., 514: 23-29.

31. Perottoni, J., L.P. Lobato, A. Silveira, J.B. Rocha and T. Emanuelli, 2004. Effects of mercury and selenite on delta-aminolevulinate dehydratase activity and on selected oxidative stress parameters in rats. Environ. Res., 95: 166-173.

32. Fujita, H., C. Nishitani and K. Ogawa, 2002. Lead, chemical porphyria, and heme as a biological mediator. Tohoku J. Exp. Med., 196: 53-64.

33. Daniell, W.E., H.L. Stockbridge, R.F. Labbe, J.S. Woods, K.E. Anderson, D.M. Bissell, J.R. Bloomer, R.D. Ellefson, M.R. Moore, C.A. Pierach, W.E. Schreiber, A. Tefferi and G.M. Franklin, 1997. Environmental chemical exposures and disturbances of heme synthesis. Environ. Health Perspect., 105 (Suppl. 1): 37-53.

34. Fowler, B.A., 2001. Porphyrinurias induced by mercury and other metals. Toxicol. Sci., 61: 197198.

35. Heyer, N.J., A.C. Bittner Jr., D. Echeverria and J.S. Woods, 2006. A cascade analysis of the interaction of mercury and coproporphyrinogen oxidase (CPOX) polymorphism on the heme biosynthetic pathway and porphyrin production. Toxicol. Lett., 161: 159-166.

36. Woods, J.S., M.A. Bowers and H.A. Davis, 1991. Urinary porphyrin profiles as biomarkers of trace metal exposure and toxicity: Studies on urinary porphyrin excretion patterns in rats during prolonged exposure to methyl mercury. Toxicol. Appl. Pharmacol., 110: 464-476.

37. Woods, J.S., C.A. Calas and L.D. Aicher, 1990. Stimulation of porphyrinogen oxidation by mercuric ion. II. Promotion of oxidation from the interaction of mercuric ion, glutathione and mitochondria-generated hydrogen peroxide. Mol. Pharmacol., 38: 261-266.
38. Woods, J.S., 1988. Attenuation of porphyrinogen oxidation by glutathione in vitro and reversal by porphyrinogenic trace metals. Biochem. Biophys. Res. Commun., 152: 1428-1434.

39. Pogribna, M., S. Melnyk, I. Pogribny, A. Chango, P. Yi and S.J. James, 2001. Homocysteine metabolism in children with Down syndrome: In vitro modulation. Am. J. Human Genet., 69: 88-95.

40. Innis, S.M., A.G.F. Davidson, A. Chen, R. Dyer, S. Melnyk and S.J. James, 2003. Increased plasma homocysteine and S-adenosylhomocysteine and decreased methionine is associated with altered phosphatidylcholine and phosphatidylethanolamine in cystic fibrosis. J. Pediatr., 143: 351-356.

41. Hobbs, C.A., M.A. Cleves, S. Melnyk, W.Z. Zhao and S.J. James, 2005. Congenital heart defects and abnormal maternal biomarkers of methionine and homocysteine metabolism. Am. J. Clin. Nutr., 81: 147-153.

42. Packer, B.R., M. Yeager, L. Burdett, R. Welch, M. Beerman, L. Qi, H. Sicotte, B. Staats, M. Acharya, A. Crenshaw, A. Eckert, V. Puri, D.S. Gerhard and S.J. Chanock, 2006. SNP500Cancer: a public resource for sequence validation, assay development, and frequency analysis for genetic variation in candidate genes. Nucleic Acids Res., 34: D617-D621.

43. Cleves, M.A., 1999. Hardy-Weinberg equilibrium tests and allele frequency estimation. STATA Technical Bull., 48: 34-37.

44. Montenegro, M.F., F. Barbosa Jr., V.C. Sandrim, R.F. Gerlach and J.E. Tanus-Santos, 2006. Ethnicity affects the distribution of deltaaminolevulinic acid dehydratase (ALAD) genetic variants. Clin. Chim. Acta, 367: 192-195.

45. Lanphear, B.P., R. Hornung, J. Khoury, K. Yolton, P. Baghurst, D.C. Bellinger, R.L. Canfield, K.N. Dietrich, R. Bornschein, T. Greene, S.J. Rothenberg, H.L. Needleman, L. Schnaas, G. Wasserman, J. Graziano, and R. Roberts, 2005. Low-level environmental lead exposure and children's intellectual function: An international pooled analysis. Environ. Health Perspect., 113: 894-899.

46. Canfield, R.L., C.R. Henderson Jr., D.A. CorySlechta, C. Cox, T.A. Jusko and B.P. Lanphear, 2003. Intellectual impairment in children with blood lead concentrations below 10 microg per deciliter. N. Engl. J. Med., 348: 1517-1526. 
47. Hu, H., M.M. Tellez-Rojo, D. Bellinger, D. Smith, A.S. Ettinger, H. Lamadrid-Figueroa, J. Schwartz, L. Schnaas, A. Mercado-Garcia and M. HernandezAvila, 2006. Fetal lead exposure at each stage of pregnancy as a predictor of infant mental development. Environ. Health Perspect., 114: 1730-1735.

48. Tellez-Rojo, M.M., D.C. Bellinger, C. ArroyoQuiroz, H. Lamadrid-Figueroa, A. MercadoGarcia, L. Schnaas-Arrieta, R.O. Wright, M. Hernandez-Avila and $\mathrm{H}$. $\mathrm{Hu}, 2006$. Longitudinal associations between blood lead concentrations lower than $10 \mathrm{microg} / \mathrm{dL}$ and neurobehavioral development in environmentally exposed children in Mexico City. Pediatr., 118: e323-e330.

49. Rice, D. and S. Barone Jr., 2000. Critical periods of vulnerability for the developing nervous system: Evidence from humans and animal models. Environ. Health Perspect., 108: 511-533.

50. Garcia-Fernandez, A.J., A.E. Bayoumi, Y. PerezPertejo, M. Motas, R.M. Reguera, C. Ordonez, R. Balana-Fouce and D. Ordonez, 2002. Alterations of the glutathione-redox balance induced by metals in CHO-K1 cells. Comp Biochem. Physiol C. Toxicol. Pharmacol., 132: 365-373.

51. Stohs, S.J. and D. Bagchi, 1995. Oxidative mechanisms in the toxicity of metal ions. Free Radic. Biol. Med., 18: 321-336.

52. Wetmur, J.G., G. Lehnert and R.J. Desnick, 1991. The delta-aminolevulinate dehydratase polymorphism: Higher blood lead levels in lead workers and environmentally exposed children with the 1-2 and 2-2 isozymes. Environ. Res., 56: 109-119.

53. Cohen, D.J., R. Paul, G.M. Anderson and D. F. Harcherik, 1982. Blood lead in autistic children. Lancet, 2: 94-95.
54. Smith, C.M., X. Wang, H. Hu and K.T. Kelsey, 1995. A polymorphism in the delta-aminolevulinic acid dehydratase gene may modify the pharmacokinetics and toxicity of lead. Environ. Health Perspect., 103: 248-253.

55. Secchi, G.C., L. Erba and G. Cambiaghi, 1974. Delta-aminolevulinic acid dehydratase activity of erythrocytes and liver tissue in man: Relationship to lead exposure. Arch. Environ. Health, 28: 130132 .

56. James, S.J., W. Slikker III, S. Melnyk, E. New, M. Pogribna and S. Jernigan, 2005. Thimerosal neurotoxicity is associated with glutathione depletion: protection with glutathione precursors. Neurotoxicology, 26: 1-8.

57. Ono, H.A. Sakamoto and N. Sakura, 2001. Plasma total glutathione concentrations in healthy pediatric and adult subjects. Clin. Chim. Acta, 312: 227-229.

58. Costa, L.G., W.F. Li, R.J. Richter, D.M. Shih, A. Lusis and C.E. Furlong, 1999. The role of paraoxonase (PON1) in the detoxication of organophosphates and its human polymorphism. Chem. Biol. Interact., 119-120: 429-438.

59. Pasca, S.P., B. Nemes, L. Vlase, C.E. Gagyi, E. Dronca, A.C. Miu and M. Dronca, 2006. High levels of homocysteine and low serum paraoxonase 1 arylesterase activity in children with autism. Life Sci., 78: 2244-2248.

60. Chamary, J.V., J.L. Parmley and L.D. Hurst, 2006. Hearing silence: Non-neutral evolution at synonymous sites in mammals. Nat. Rev. Genet., 7: $98-108$. 